\title{
Hypersensitivity Reactions to Biologicals: an EAACI position
} paper

Sevim Bavbek ${ }^{1}$, Mauro Pagani ${ }^{2}$, Emilio Alvarez-Cuesta ${ }^{3}$, Mariana Castells ${ }^{4}$, Adile Berna Dursun $^{5}$, Sahar Hamadi ${ }^{6}$, Ricardo Madrigal-Burgaleta ${ }^{7}$, Soledad Sanchez-Sanchez ${ }^{8}$, and Alessandra Vultaggio ${ }^{9}$

${ }^{1}$ Ankara University Faculty of Medicine

${ }^{2}$ ASST Mantova

${ }^{3}$ Hospital Ramón y Cajal

${ }^{4}$ Brigham and Women's Hospital

${ }^{5}$ Recep Tayyip Erdoğan University

${ }^{6}$ Division of Immunology and Allergy; Department of Medicine, Brigham and Women's

Hospital

${ }^{7}$ St Bartholomew's Hospital, Barts Health NHS Trust

${ }^{8}$ University Hospital Complex A Coruña (CHUAC),

${ }^{9}$ Azienda Ospedaliera Universitaria Careggi,

March 31, 2021

\begin{abstract}
Because of their selectivity, biologicals are crucial therapeutic agents in oncological, immunological, and inflammatory diseases and their use in clinical practice is broadening. Biologicals are among the most common drugs that can cause hypersensitivity reactions (HSRs), and this is primarily attributed to an explosion in new treatment options that has developed through personalized and precision medicine. Patients can develop HSRs to these agents during the first lifetime exposure or after repeated exposure. Despite its relatively high prevalence, the underlying mechanisms and optimal management of HSRs to biologicals remain incompletely explained. In this position paper, the authors provided evidence-based recommendations for the diagnosis and management of HSRs to biologicals. Additionally, the document defines unmet needs, which should be topics of future studies.
\end{abstract}

\section{Hosted file}

Complete manuscript with Tables and Figure Hypersensitivity Reactions to Biological Agents.pdf available at https://authorea.com/users/405076/articles/516110-hypersensitivity-reactionsto-biologicals-an-eaaci-position-paper 